\title{
Object-based connectedness facilitates matching
}

\author{
ARNO KONING and ROB VAN LIER \\ University of Nijmegen, Nijmegen, The Netherlands
}

\begin{abstract}
In two matching tasks, participants had to match two images of object pairs. Image-based (IB) connectedness refers to connectedness between the objects in an image. Object-based (OB) connectedness refers to connectedness between the interpreted objects. In Experiment 1, a monocular depth cue (shadow) was used to distinguish different relation types between object pairs. Three relation types were created: IB/OB-connected objects, IB/OB-disconnected objects, and IB-connected/OB-disconnected objects. It was found that IB/OB-connected objects were matched faster than IB/OB-disconnected objects. Objects that were IB-connected/OB-disconnected were matched equally to IB/OB-disconnected objects. In Experiment 2, stereoscopic presentation was used. With relation types comparable to those in Experiment 1, it was again found that $\mathrm{OB}$ connectedness determined speed of matching, rather than IB connectedness. We conclude that matching of projections of three-dimensional objects depends more on OB connectedness than on IB connectedness.
\end{abstract}

In order to recognize objects, as well as parts of objects, the visual system has to segment proximal stimulations. This segmentation often proceeds at boundaries or edges. Several factors play an important role in this segmentation process. Properties such as luminance (e.g., Agostini \& Proffitt, 1993), texture (e.g., Beck, 1982), and color (e.g., Fuchs, 1923; van Lier \& Wagemans, 1997) influence the segmentation of objects and parts of objects, but also the form of the object to be segmented is important. In describing the segmentation process, several approaches in visual perception have often relied on deep concavities between objects (Baylis \& Driver, 1993; Biederman, 1987; Hoffman \& Richards, 1984; Hoffman \& Singh, 1997; Siddiqi, Tresness, \& Kimia, 1996), but not so much on connectedness between objects.

Segmentation is mediated by grouping principles, such as the Gestalt principles of similarity, symmetry, and proximity. Of these principles, proximity bears a close relation to connectedness. Generally, the closer two objects are to each other, the more likely it is that they will be grouped together (e.g., Kubovy, Holcombe, \& Wagemans, 1998; Kubovy \& Wagemans, 1995; Wertheimer, 1923). Connectedness between visual elements can be considered as an extreme form of proximity. Note that, on a somewhat different basis, Palmer and Rock (1994) defined the principle of uniform connectedness. Uni-

\footnotetext{
Parts of this study were presented at the first annual meeting of the Vision Sciences Society, which was held in Sarasota, FL (2001). R.v.L. received a grant from the Royal Netherlands Academy of Arts and Sciences. This research was conducted at the Nijmegen Institute for Cognition and Information. The authors thank Johan Wagemans, Peter van der Helm, Tessa de Wit, Marco Puts, Emanuel Leeuwenberg, and two anonymous reviewers for their helpful comments and suggestions on a previous draft of this article. Correspondence concerning this article should be addressed to A. Koning, Room B.01.25, NICI, University of Nijmegen, P.O. Box 9104, 6500 HE Nijmegen, The Netherlands (e-mail: a.koning@nici.kun.nl).
}

form connectedness is a special case of connectedness in which two connected regions, sharing the same visual properties, serve as a single perceptual entry-level unit, being the units with which further grouping and segmentation processes start.

Connectedness between shapes is crucial in determining the representational unity of two or more objects, and it influences grouping of shapes and objects, both on an early level (Palmer \& Rock, 1994) and on subsequent levels of perception (Saiki \& Hummel, 1998; van Lier \& Wagemans, 1998). Using a rapid serial visual presentation paradigm, Saiki and Hummel found a facilitation for the recognition of connected shapes, as compared with disconnected shapes. More specifically, Saiki and Hummel showed that connected target shapes were easier to distinguish among a set of distractors, as compared with disconnected target shapes. They suggested that the visual system groups parts into object-based sets on the basis of image connectedness. Similarly, van Lier and Wagemans (1998) investigated the effect of connectedness between two-dimensional (2-D) shapes. Their stimuli consisted of multipart configurations of 2-D shapes in which the shapes could be either connected to each other or disconnected from each other. Using a matching paradigm, van Lier and Wagemans (1998) found faster reaction times (RTs) for connected 2-D shapes, as compared with disconnected 2-D shapes, when mental rotation was required. Van Lier and Wagemans (1998) suggested that this indicated a higher representational unity for connected shapes than for disconnected shapes.

The results above cannot simply be extended to threedimensional (3-D) objects. When 3-D objects are projected, image connectedness between these objects does not necessarily imply real world connectedness between them, as Saiki and Hummel (1998) have already noted. An accurate judgment as to whether or not objects are connected in 3-D space cannot be made if this judgment 
is based solely on their connectedness in the image. Additional environmental cues, such as, for example, shadows, are needed to make an appropriate judgment (see, e.g., Kersten, Mamassian, \& Knill, 1997; Madison, Thompson, Kersten, Shirley, \& Smits, 2001; Meng \& Sedgwick, 2001). As an example, two boxes are shown in Figure 1A. It can be seen here that the relation between these boxes in 3-D space is ambiguous. One object partly occludes the other, but in the image there are no additional cues available to the perceiver to determine whether or not the two boxes are actually connected to each other in 3-D space. The same two boxes are shown in Figure 1B. The 2-D relation between the two boxes is identical to that in Figure 1A. However, due to the shadow of the small box on the large box, the ambiguity of the spatial relation between the boxes is now resolved. The small box is seen as floating in front of the large box. Apparently, a relatively simple cue, such as a shadow, can disambiguate the connectedness in 3-D space between objects.

When 3-D objects are used, a distinction can thus be made between image-based (IB) connectedness and object-based (OB) connectedness. Here, IB connectedness refers to the connectedness between 3-D objects as they appear in the image. Note that IB connectedness, as it is described here, differs from uniform connectedness in that IB connectedness does not require homogeneous regions between two connected shapes or objects. In this respect, IB connectedness may be seen as a weaker form of connectedness than is uniform connectedness. OB connectedness refers to the connectedness between objects in 3-D space. That is, OB connectedness refers to the connectedness of the interpreted distal objects. An image of two 3-D objects can show these objects to be both IB and OB connected. Likewise, two 3-D objects can be both IB and OB disconnected. An image of 3-D objects can also contain two objects that are IB connected but OB disconnected (as in Figure 1B). In this case, there is an incongruency between IB and OB connectedness in which the additional depth cue (shadow) can disambiguate the image. In line with this, Meng and
Sedgwick (2001) reported a series of experiments in which they investigated how well human observers were able to use monocular depth cues in judgments of distance perception. Participants had to judge the distance of a cube that rested on a platform, which, in turn, could be resting on the ground. Meng and Sedgwick called such interobject relations nested contact relations. They found that such nested contact relations could effectively be used by participants to make accurate judgments. In addition, Meng and Sedgwick showed that when a monocular depth cue (shadow) was provided, participants were able to use this information to make correct judgments. They concluded that the perceived distance of an object can be mediated by another object, provided that there is a clear relation, such as connectedness, between the objects (Meng \& Sedgwick, 2001).

In this research, we use both monocular and binocular depth cues to investigate the relative role of IB connectedness and OB connectedness. In Experiment 1, we used monocular depth cues (shadows) to include the abovementioned case in which there is an incongruency between IB and OB connectedness. In Experiment 2, we used binocular depth cues (stereoscopic presentation) to further investigate connectedness between 3-D objects. Analogous to van Lier and Wagemans (1998), the matching paradigm was used here. In Experiment 1, three relation types were used: IB/OB connected, IB/OB disconnected, and IB-connected/OB-disconnected. As the type of concavity between two shapes also influences the strength of the connectedness between them (Hoffman \& Singh, 1997; van Lier \& Wagemans, 1998), we additionally manipulated the concavities between pairs of objects. Acute and obtuse concavities between $3-\mathrm{D}$ objects were used here.

\section{EXPERIMENT 1}

\section{Method}

Participants. Twenty participants (ages, 20-28 years) were given course credit or were paid for their time. All the participants had normal or corrected-to-normal vision.

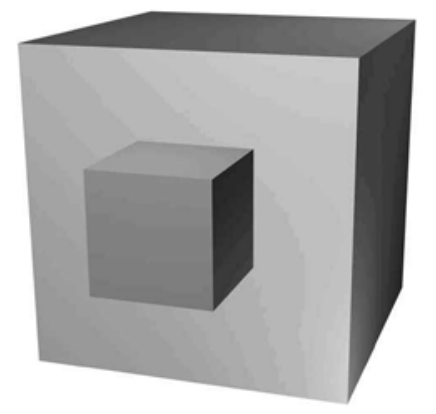

(A)

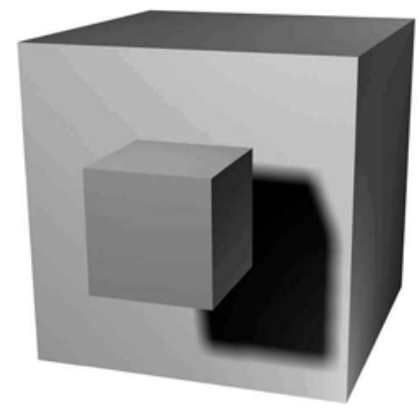

(B)

Figure 1. (A) An example of ambiguous object-based (OB) connectedness.

(B) An example of disambiguated $O B$ connectedness by means of a shadow. 
Stimuli. Each image contained two 3-D objects-that is, one large object and one small object. Three different large objects and three different small objects were created (using a 3-D modeling software package, 3-D Studio Max R2, Autodesk, Inc.; see Figure 2).

The small objects consisted of a tapered cylinder (a fez), a prism, and a pyramid. The large objects were derived from a box, a cylinder, and an extruded hexagon, respectively, and were tapered to make them asymmetrical with respect to the horizontal plane. The two objects in an image could be related to each other in three different ways (see Figure 3). To establish these three relations, the objects were moved with respect to each other. Also, by using a spotlight, a shadow of the small object on the large object was added to disambiguate the $\mathrm{OB}$ connectedness between the objects.

In the first relation type, the touch relation, the two objects were connected to each other on both the IB level and the OB level (see Figure $3 \mathrm{~A}$ ). In the second relation type, the loose relation, the two objects were separated from each other on both the IB level and the OB level (see Figure 3B). In the third relation type, the float relation, the objects were connected on the IB level but disconnected from each other on the OB level (see Figure 3C). Both the large and the small objects could be oriented in two ways, upright or upside down. Upright referred to the situation in which the smallest part of an object was facing upward, while the largest part was facing downward. The orientation of the small objects resulted in different concavities between the small and the large objects (acute concavity when the angle between the objects was $<90^{\circ}$ and obtuse concavity when the angle between the objects was $>90^{\circ}$ ).

A same-different matching task was used in which it was the participant's task to judge the similarity of two simultaneously presented images. Each trial consisted of two images that were positioned side by side. The images could be identical to each other or not. Half of the trials were matches; the other half were nonmatches. The nonmatch trials were created by systematically changing only the orientation of one object or both objects. The relation type and the types of objects were kept the same within each trial. All the images were also rotated $180^{\circ}$. Consequently, the rotation variable refers to the orientation of the images in a trial. Two images could have either the same orientation or a different orientation in a trial.

A total of 864 trials were used: three small objects, two possible orientations for each small object, three large objects, two possible orientations for each large object, three relation types, two same orientations of images in a trial, two different orientations of images in a trial, and match/nonmatch trials. A practice task was also created, using different objects, to familiarize the participant with the experimental setup and the procedure.

Procedure. The experiment was conducted at the University of Nijmegen. Lighting conditions (dimly lit) were kept constant across all participants. The trials were presented on a 15 -in. monitor at a resolution of $800 \times 600$ pixels, controlled by an IBM-compatible computer with Windows 98 and an Intel Pentium II processor. The participants were seated about $1 \mathrm{~m}$ from the screen. The two images covered an area of $18.5 \times 5.8 \mathrm{~cm}$ on the screen (i.e., a visual angle of about $11^{\circ} \times 3^{\circ}$ for a single display). The software used was SuperLab Pro (Cedrus, Inc.). First, the task was explained to the participant. After examples of both a match trial and a nonmatch trial were shown, the practice task was started. A response box (Cedrus, Inc.) with 1-msec accuracy was used.

A fixation cross $(750 \mathrm{msec})$ preceded each trial. The trial remained on the screen until a response was given. It was the participants task to compare the two images and decide, as quickly as possible, whether or not they were the same, regardless of the orientation of the images. A label below each button indicated either the same or the different response. During the practice task, visual feedback was given on the monitor as to whether or not the response was correct. In the main task, no feedback was given. Instead, a blank screen was presented, and after $750 \mathrm{msec}$, the fixation cross appeared again, and the next trial was started. The practice task consisted of 30 trials and took about 5 min to complete. Next, the experiment was started, which took about $50 \mathrm{~min}$ to complete. All 864 trials of the main task were presented randomly to each participant in a single session. The buttons on the response box were balanced left-right across participants.

\section{Results}

There was a $5.5 \%$ error rate over all participants for the match trials. There was no speed-accuracy tradeoff. Analyses were done on the correct match trials. We measured RT as the dependent variable in a three-factorial design, rotation $(2) \times$ relation type $(3) \times$ concavity $(2)$. $A$ repeated measures analysis of variance (ANOVA) yielded three significant main effects. A main effect was found for the rotation variable $[F(1,19)=66.88, p<.001]$. Trials in which no mental rotation was necessary were matched faster $(M=898.68 \mathrm{msec}, S E M=11.56)$ as compared with trials in which mental rotation was required $(M=1,279.65 \mathrm{msec}, S E M=20.83)$. A second main effect was found for the relation type variable $[F(2,18)=$ 5.24, $p<.05$; see Figure 4).

The images in the touch relation were matched faster than the images in the float relation and the images in the loose relation. Contrast comparisons revealed a signifi-

\section{4}
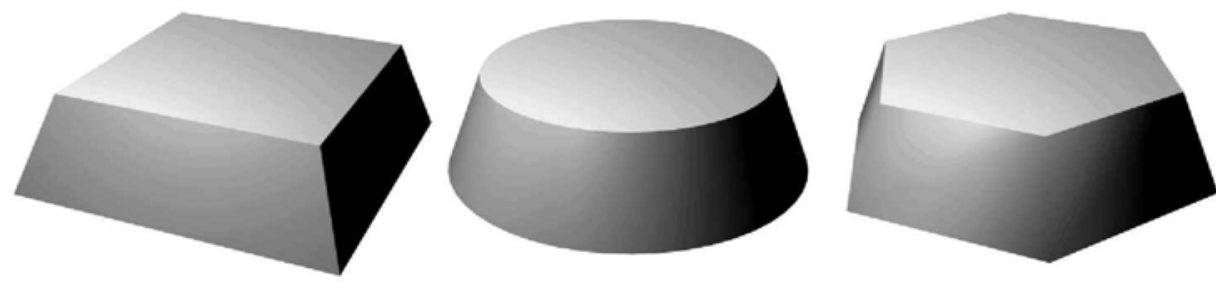

Figure 2. Experiment 1 : examples of the large and small objects used. From left to right and from top to bottom: tapered cylinder ( $f e z)$, prism, pyramid, box, cylinder, and hexagon. 


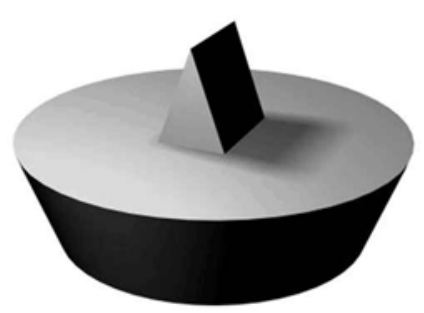

(A)

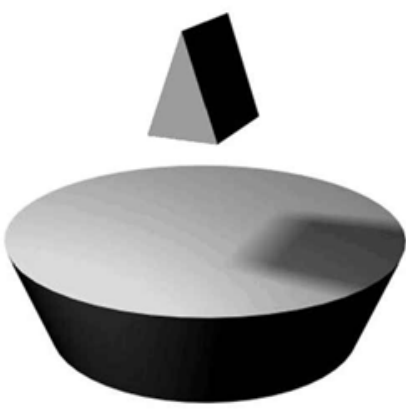

(B)

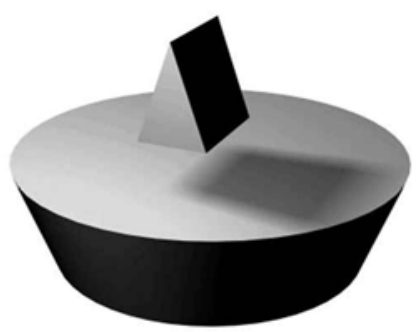

(C)

Figure 3. Experiment 1: examples of the three relation types used. (A) Touch relation. (B) Loose relation. (C) Float relation.

cant difference between the touch relation and the float relation $[F(1,19)=9.79, p<.01]$, and between the touch relation and the loose relation $[F(1,19)=7.23, p<.05]$. There was no significant difference between the float relation and the loose relation $(F<1)$. A third main effect was found for the concavity variable $[F(1,19)=25.59$, $p<.001]$. Pairs of images that showed an obtuse concavity between the two objects were matched faster $(M=$ $1,053.81 \mathrm{msec}, S E M=15.38)$ than pairs of images that showed an acute concavity between the objects $(M=$ $1,124.51 \mathrm{msec}, S E M=16.79)$. An interaction was found between rotation and concavity $[F(1,19)=5.74, p<.05]$. Overall, obtuse concavities were matched faster than acute concavities. This difference between the two concavities was larger when the objects had to be rotated. A second interaction was found between relation type and concavity $[F(2,18)=3.76, p<.05]$. In the case of acute concavities, there were no significant differences between the three relations (all $p \mathrm{~s}>.2$ ). In the case of obtuse concavities, matching was fastest in the touch relation, as compared with the float relation $[F(1,19)=$ $19.33, p<.001]$ and the loose relation $[F(1,19)=12.65$, $p<.005]$. There was no significant difference between the float relation and the loose relation $(F<1)$.

\section{Discussion}

The rotation variable yielded a highly significant main effect. Two images with the same orientation were matched faster than pairs of images that had a different orientation with respect to each other. This is in line with previous studies on mental rotation (e.g., Shepard \& Metzler, 1971). The second main effect was found for the relation type variable. When objects are connected on both the IB and the OB levels, or when objects are disconnected on both levels, the results are comparable to those found in studies with 2-D shapes (van Lier \& Wagemans, 1998). In the crucial relation type, the float relation, no differences were found in RTs, as compared with the images in the loose relation, even though there is connectedness between the objects in the float relation on the IB level. This suggests that the images in the float relation resemble images of pairs of completely disconnected objects more than they resemble images of pairs of completely connected objects, at least on a perceptual level. Apparently, OB connectedness is the determining factor for the matching of 3-D objects.

The third main effect that was found was for the concavity variable. There was an advantage for obtuse, as compared with acute, concavities. Note that even though there was neither an IB connectedness nor an OB connectedness between the objects in the loose relation, matching was still faster when the orientation of the small object was upright, as compared with upside down. That is, the matching of these pairs of objects was faster when the "connectedness" between the objects inclined toward obtuse, as compared with when the "connectedness" inclined toward acute. An interaction was found between rotation and concavity as well as between rela-

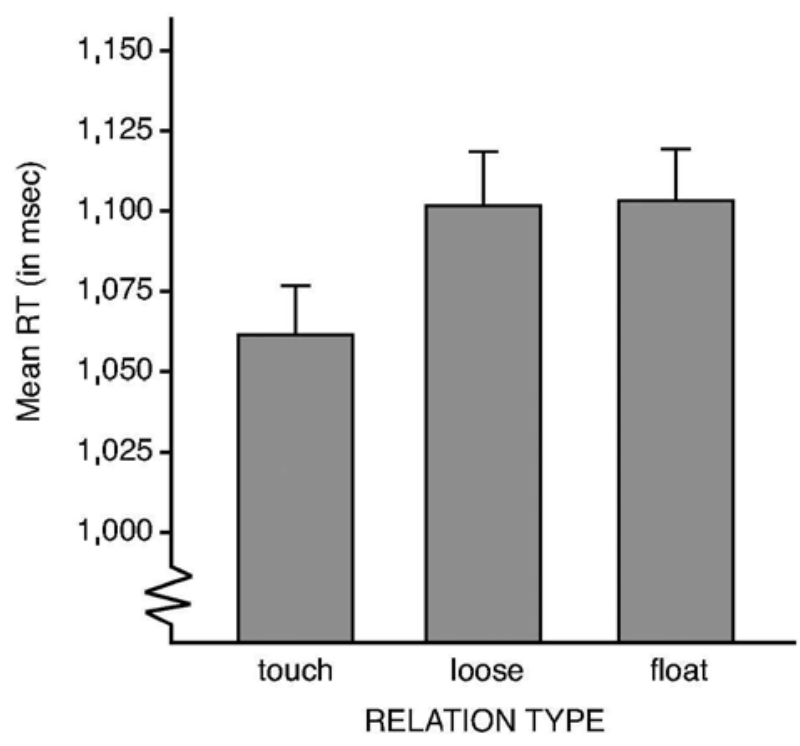

Figure 4. Experiment 1: graph of reaction time (RT), with $S E M$ error bars, as a function of the relation type variable. 
tion type and concavity. The results regarding the concavity variable can be explained in terms of part salience, as presented by Hoffman and Singh (1997). Hoffman and Singh suggested that the salience of a part boundary increases as the degree of concavity increases (obviously, acute connections show deeper concavities than do obtuse concavities). In terms of Hoffman and Singh, the acute connections that were used here may then have resulted in more salient parts than did the obtuse connections. One may then say that the obtuse concavities between the objects revealed a stronger connection than did the acute concavities. In addition, the obtuse concavities all showed plane-to-plane connections between the small and the large objects, whereas the acute concavities also showed edge-to-plane connections and point-to-plane connections. This additional difference possibly may have strengthened the effect of concavity. Further implications of the concavity variable are discussed in a later section.

To summarize, the first experiment provided evidence that matching of 3-D objects depends on their connectedness in 3-D space, regardless of their connectedness in the image itself. The result that stands out most is that the matching of images in the float relation appears to resemble the matching of images in the loose relation, but not the matching of images in the touch relation. Even though the 3-D percept of the images was quite compelling, the shadows were the only additional monocular depth information. The shadows were added to create the distinction between the touch relation and the float relation, but they might have been interpreted differently than was intended and, therefore, might have influenced the results. For example, although it was explained to the participant that shadows were present, the detached shadows in Figures 3B and 3C might have appeared to be a third entity, whereas the shadow in Figure $3 \mathrm{~A}$ appeared to be tightly bound to the small object.

In the second experiment, we created comparable relation types without using shadows. Instead, we used stereoscopic presentation as an alternative tool to create the perception of depth and, hence, disconnectedness on the basis of binocular depth cues.

\section{EXPERIMENT 2}

Again, a same-different matching task was used. The relation types that were used were identical to the touch relation and the float relation in Experiment 1 and were created by making use of different depth layers. We will focus only on the touch relation and the float relation, since the significant differences between the RTs in these conditions in Experiment 1 were most striking. Moreover, including a loose relation in Experiment 2 would lead to very different stimuli in which IB-disconnected objects would also be perceived in a different depth layer. To control for the introduction of different depth layers, two touch relations were created: one in which no depth would be perceived (called the touch-I relation) and one in which both objects would be perceived in front of the screen (the touch-II relation). In both the touch-I relation and the touch-II relation, the objects were IB and OB connected. The third relation type is referred to as the float relation. The objects in this relation type were IB connected but OB disconnected (due to the perception of depth, created by presenting images stereoscopically). Again, it was expected that performed matching would be based on OB connectedness. Therefore, no differences were expected to be found between the two touch relations. Since the objects in the float relation were OB disconnected, these images were expected to be matched more slowly than the touch-I and touch-II relations.

\section{Method}

Participants. Thirteen participants (ages, 24-33 years), who did not participate in the first experiment, were given course credit or were paid for their time. All the participants had normal or corrected-tonormal vision.

Stimuli. Stimulus construction started with two simple geometric shapes (again using 3-D Studio Max; see Figure 5). There were various ways in which positional aspects were accounted for. One of the objects was oriented horizontally, whereas the other was oriented vertically. Both objects could be positioned either in the front or in the back. In addition, the horizontally oriented object could be positioned at the top or at the bottom of the image and could have its smaller side pointing to the left or to the right. The vertically oriented object could be positioned on the left side or the right side of the image, and it could have its smaller part pointing upward or downward. With these variations, a total of 32 object configurations were created. Of these 32 object configurations, 16 were $180^{\circ}$-rotated versions of the other 16 .

For each of the configurations, a stereoscopic image pair was made. All the objects in an image were presented on a white background. In all pairs of images, there was a $3^{\circ}$ angular rotational difference between the objects in the images around the vertical object axis. Three different relation types were created by slightly changing the horizontal positions of the objects. Images in the touch-I relation consisted of two otherwise identical images (see Figure 5A). When fused, these images did not show an additional depth layer. With respect to the touch-II relation, both objects in the right-eye image were positioned slightly to the left ( 5 arcmin). Consequently, when fused, both objects were perceived in front of the white background (see Figure 5B). With respect to the float relation, the front object in the right-eye image was positioned slightly to the left, whereas the front object in the left-eye image was positioned the same distance to the right. The total range of disparity was 5 arcmin, similar to the touch-II relation (see Figure 5C). Consequently, when fused, the front object was perceived as floating in front of the rear object. In the float relation, the front object was repositioned in both images, instead of in just one image. This was done to minimize the change in 2-D overlap between the float relation and both touch relations when the images were fused.

A same-different matching task, similar to that in Experiment 1, was used, for which a total of 384 trials were created: 32 object configurations, three types of connectedness, rotation/no-rotation, and match/nonmatch trials.

Procedure. The procedure was identical to that in the first experiment, with the following exceptions. The experiment was performed in a completely darkened room. The trials were presented on a 19-in. monitor at a resolution of 1,024 $\times 768$ pixels, controlled by an IBM-compatible computer with an Intel Pentium III processor. The participant's head was stabilized by a chinrest. A standard double-mirror setup was used. The visual path (including the bouncing off the mirrors) between the screen and the participant's 

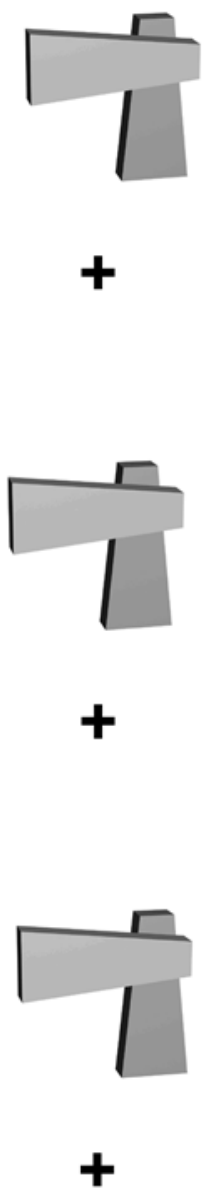

$+$

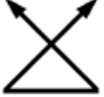

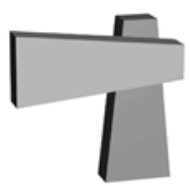
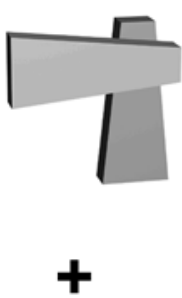

(A)
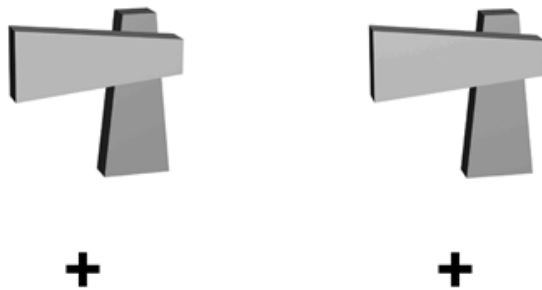

(B)
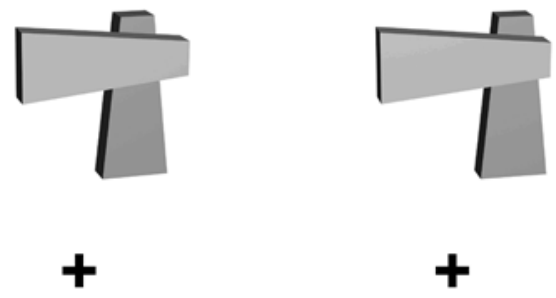

(C)

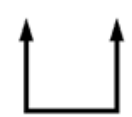

Figure 5. Experiment 2: examples of the objects used. For all relation types, there was a $3^{\circ}$ angular rotational difference between the objects in the images around the vertical object axis. Each pair of images can be fused by crossed convergence (left column and center column) or by uncrossed convergence (center column and right column), to obtain the effects of object-based (dis)connectedness. (A) Example of images in the touch-I relation. When fused, these images do not show an added depth layer. (B) Example of images in the touch-II relation. When fused, both objects are perceived in front of the background. (C) Example of images in the float relation. When fused, the front object is perceived as floating in front of the rear object. See the text for details.

eyes was $127 \mathrm{~cm}$. Two images were presented in the upper half of the screen, and two images were presented in the lower half of the screen. The mirrors were positioned in such a manner that the images in the upper half of the screen were fused to form one image, and likewise for the images in the lower half of the screen. As a result, in each trial, one image was presented above and one was presented below the position of the fixation cross. The images were presented on a black background. When fused, one image covered an area of $5.5 \times 5.5 \mathrm{~cm}$ on the screen (i.e., a visual angle of less than $3^{\circ}$ ). The contrast of the black background of the screen with the white background of the images provided a means to control whether fusion was stabilized. For a single display in which the images were fused, the visual angle spanned less than $3^{\circ}$ horizontally and less than $7^{\circ}$ vertically.

Prior to the practice task, a test screen was presented in which three images were presented. These images showed different objects but the same relation types as those used in the experiment. The participants were asked whether they saw three pictures and whether they saw any differences regarding depth in the images. Only when the participant reported seeing differences in depth between the touch-I, the touch-II, and the float relations was the practice task started. This was done to stabilize fusion and to see 
whether the participants could actually see the differences in depth. The task took about 30 min to complete.

\section{Results}

There was a $3.9 \%$ error rate over all participants for the match trials. There was no speed-accuracy tradeoff. The analyses were done on the correct match trials in a two-factorial design, relation type $(3) \times$ rotation $(2)$, with RT as the dependent variable. In a repeated measures ANOVA, a main effect was found for relation type $[F(2,11)=5.27, p<.05]$. Contrast comparisons revealed that the float relation differed significantly from the touch-I relation $[F(1,12)=11.33, p<.01]$ and also from the touch-II relation $[F(1,12)=4.88, p<.05]$. The touch-I and the touch-II relations did not differ from each other $(F<1)$. The rotation variable also showed a significant main effect $[F(1,12)=109.954, p<.001]$. Trials in which no mental rotation was required were matched faster $(M=1,535.58 \mathrm{msec}, S E M=36.64)$ than trials in which mental rotation was required $(M=$ 2,709.73 msec, $S E M=58.11)$. The interaction between the two variables was not significant $(F<1$; see Figure 6 for a graph of the main effect of relation type).

\section{Discussion}

As in the first experiment, the rotation variable yielded a significant main effect. A significant main effect was also found for relation type. Objects in the touch-I relation were matched faster than objects in the float relation. Since in the touch-I relation there was no additional depth information, another relation type was added: the touch-II relation. Objects in this relation type were also IB and OB connected, but both objects were clearly seen as floating in front of the white background. The RTs for this relation type also differed significantly

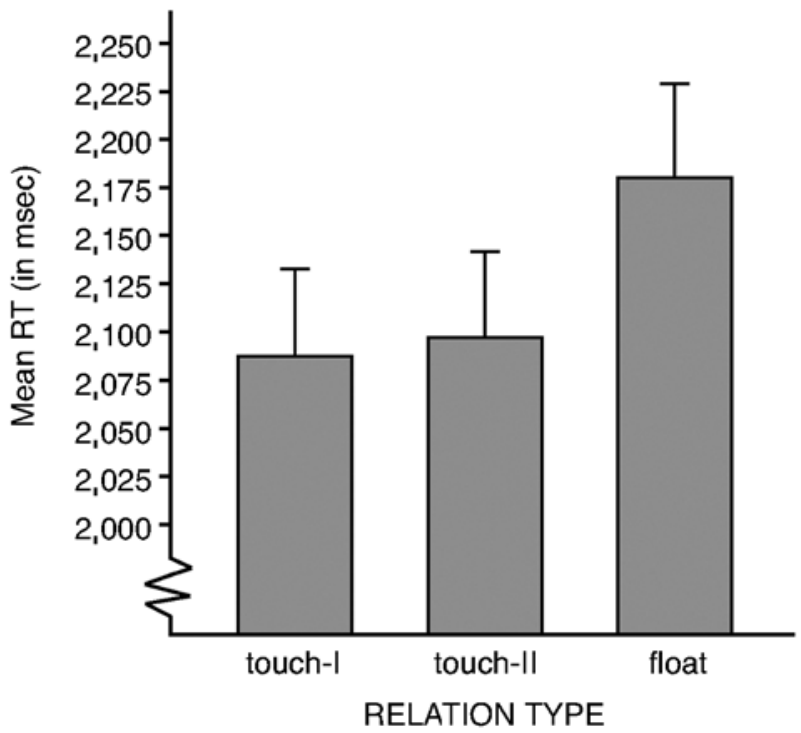

Figure 6. Experiment 2: graph of reaction time (RT), with $S E M$ error bars, as a function of the relation type variable. from the RTs for the float relation. The results on the two touch relations did not differ from each other. These findings strengthen our previous finding in Experiment 1 that matching depends on OB connectedness. The added depth layer in the touch-II relation did not influence the matching of objects, as compared with the touch-I relation.

\section{GENERAL DISCUSSION}

Pairs of 3-D objects that are connected in 3-D space are matched faster than pairs of objects that are disconnected in 3-D space. This finding extends earlier work done on 2-D shape matching (van Lier \& Wagemans, 1998) to the stimulus domain of 3-D objects. In the first experiment, it was found that the float relation images showed the same RT results as the loose relation images even though the float relation images seemed more similar to the touch relation images. We therefore conclude that the OB connectedness between the objects was the determining factor in the speed of object matching. In the second experiment, this finding was verified by means of a different operationalization. By using stereoscopic presentation, OB connectedness was varied without the use of shadows. The results show that the speed of matching of pairs of objects depended on the OB connectedness between the objects. The present results will now be discussed with respect to some related research.

Saiki and Hummel (1998) performed several experiments in which they looked at various types of connectedness and part-relation integrations between 2-D shapes and filled line drawings of 3-D objects. Some differences and similarities between the two studies are to be mentioned here. Saiki and Hummel used a rapid serial visual presentation paradigm in which a target was to be identified among a number of distractors. In our study, a matching paradigm was used instead. In contrast with Saiki and Hummel, we used mental operations, such as translation and rotation. Nevertheless, our findings are compatible with those of Saiki and Hummel. In their Experiment 6, Saiki and Hummel examined different depth layers (i.e., coplanar vs. partly occluded objects). They suggested that image connectedness is neither necessary nor sufficient for part-relation integration. By means of both studies, it has been established that not IB connectedness but, rather, OB connectedness is most effective. In addition, we used both monocular and binocular depth cues in distinguishing the different types of connectedness. The fact that agreement between the two studies has come forth from different experimental paradigms further strengthens the conclusions drawn.

The present results can perhaps be explained by the phenomenon of OB attention. The objects in the touch relations could have been perceived more as one object than as two objects. Previous research (Duncan, 1993; Kahneman, Treisman, \& Gibbs, 1992) has shown that attending to one object is easier than attending to two objects. In our experiments, such a one-object advantage may have led to faster RTs for the touch relations than 
for the other relation types. However, it is unclear to what extent connectedness as such plays a mediating role in OB attention. Kramer and Watson (1996), for example, found that if two visual properties have to be searched for, there is a one-object advantage when these visual properties share the same uniform connected region. In contrast, they did not find such an advantage when these visual properties did not share the same uniform connected region (Kramer \& Watson, 1996). Whether an account of OB attention would be a suitable framework or not, the relevant issue here is a different one, since it concerns the relative role of IB and OB connectedness.

In addition to the measured effects of connectedness, the degree of concavity appeared to be an important factor as well. Recall that in Experiment 1, faster RTs were found for obtuse concavities than for acute concavities. Although the effects of obtuse and acute concavities may be explained by the notion of part salience (Hoffman \& Singh, 1997), the measured effects of the depth cues cannot be explained directly by the notion of part salience. After all, parsing at deep concavities, as described by Hoffman and Richards (1984) and further elaborated by Hoffman and Singh, depends on the presence of local junctions between objects in an image. Yet, in both Experiment 1 and Experiment 2, the local junctions between the objects remained the same when the touch relations and the float relations were compared. Although the effects of depth cues may not be explained by the notion of part salience, OB disconnectedness may increase part salience. Furthermore, Peterson (1994) has argued that in the case of depth perception, the visual system cannot rely on early processes, such as the notion of part salience, alone. Instead, the outputs of representations of objects, among others, need to be taken into account (Peterson, 1994). This is in agreement with our finding that connections between objects alone (i.e., edge extraction) does not suffice to distinguish $\mathrm{OB}$ connectedness from OB disconnectedness.

In the present experiments, IB connectedness was combined with OB connectedness, and three relation types were derived from the combination of these two factors. Obviously, a fourth relation type is possible. This relation type would show an object that is IB disconnected and $\mathrm{OB}$ connected. Creation of such an object is possible, for example, by using occlusion as an independent variable (see, e.g., Behrmann, Zemel, \& Mozer, 1998 ) or by using illusory surfaces. Concerning the latter, one might, for example, consider an object (say, a cylinder) of which the middle part is "occluded" by an illusory rectangle. Now, the remaining object parts are disconnected at the image level, but they still belong to the same object. However, we did not include such stimuli, since the interpretation of the experimental data would be less straightforward in terms of IB/OB connectedness, because of possible influences of other aspects dealing with illusory surfaces or amodal completion.

All in all, the experiments reported here lead to the suggestion that, in object recognition, an OB approach to the matching of 3-D objects needs to be taken. Much research has been done regarding differential effects of IB and OB properties. This research has included Bayesian modeling (Liu \& Kersten, 1998), the role of vertices with respect to different objects (Pilon \& Friedman, 1998), ideal observers (Tjan \& Legge, 1998), and the search for specific object properties (Watson \& Kramer, 1999). These studies should ultimately have their impact on models of object recognition (e.g., Biederman, 1987; Tarr, 1995), in that such models should be able to account for the relative influences of various types of connectedness.

\section{CONCLUSION}

The findings reported here give an insight into the concept of connectedness, both on the image-based level and on the object-based level of 3-D objects. We conclude that in the matching of 3-D objects, OB connectedness seems to be more important than IB connectedness.

\section{REFERENCES}

Agostini, T., \& Proffitt, D. (1993). Perceptual organization evokes simultaneous lightness contrast. Perception, 22, 263-272.

Baylis, G. C., \& Driver, J. (1993). Visual attention and objects: Evidence for hierarchical coding of location. Journal of Experimental Psychology: Human Perception \& Performance, 19, 451-470.

BECK, J. (1982). Textural segmentation. In J. Beck (Ed.), Organization and representation in perception (pp. 285-318). Hillsdale, NJ: Erlbaum.

Behrmann, M., Zemel, R. S., \& Mozer, M. C. (1998). Object-based attention and occlusion: Evidence from normal participants and a computational model. Journal of Experimental Psychology: Human Perception \& Performance, 24, 1011-1036.

Biederman, I. (1987). Recognition-by-components: A theory of human image understanding. Psychological Review, 94, 115-147.

Duncan, J. (1993). Similarity between concurrent visual discriminations: Dimensions and objects. Perception \& Psychophysics, 54, 425-430.

FucHS, W. (1923). Experimentelle Untersuchungen über die Änderung von Farben unter dem Einfluss von Gestalten (Angleichungserscheinungen) [Experimental investigations on the alteration of color under the influence of gestalts]. Zeitschrift für Psychologie, 92, 249-325.

Hoffman, D. D., \& Richards, W. A. (1984). Parts of recognition. Cognition, 18, 65-96.

Hoffman, D. D., \& Singh, M. (1997). Salience of visual parts. Cognition, 63, 29-78.

Kahneman, D., Treisman, A., \& GibBs, B. (1992). The reviewing of object files: Object-specific integration of information. Cognitive Psychology, 24, 175-219.

Kersten, D. J., Mamassian, P., \& Knill, D. C. (1997). Moving cast shadows induce apparent motion in depth. Perception, 26, 171-192.

Kramer, A. F., \& Watson, S. E (1996). Object-based visual selection and the principle of uniform connectedness. In A. F. Kramer, M. G. H. Coles, \& G. D. Logan (Eds.), Converging operations in the study of visual selective attention (pp. 395-414). Washington, DC: American Psychological Association.

Kubovy, M., Holcombe, A. O., \& Wagemans, J. (1998). On the lawfulness of grouping by proximity. Cognitive Psychology, 35, 71-98.

Kubovy, M., \& WAGEMANS, J. (1995). Grouping by proximity and multistability in dot lattices: A quantitative Gestalt theory. Psychological Science, 6, 225-234.

Liu, Z., \& Kersten, D. (1998). 2D observers for human 3D object recognition? Vision Research, 38, 2507-2519.

Madison, C. M., Thompson, W., Kersten, D., Shirley, P., \& Smits, B. (2001). Use of interreflection and shadow for surface contact. Perception \& Psychophysics, 63, 187-194. 
Meng, J. C., \& Sedgwick,H. A. (2001). Distance perception mediated through nested contact relations among surfaces. Perception \& Psychophysics, 63, 1-15.

Palmer, S. E., \& Rock, I. (1994). Rethinking perceptual organization: The role of uniform connectedness. Psychonomic Bulletin \& Review, 1, 29-55.

Peterson, M. A. (1994). The proper placement of uniform connectedness. Psychonomic Bulletin \& Review, 1, 509-514.

Pilon, D. J., \& Friedman, A. (1998). Grouping and detecting vertices in 2-D, 3-D, and quasi-3-D objects. Canadian Journal of Experimental Psychology, 52, 114-127.

SAIKI, J., \& Hummel, J. E. (1998). Connectedness and the integration of parts within relations in shape perception. Journal of Experimental Psychology: Human Perception \& Performance, 24, 227-251.

Shepard, R. N., \& Metzler, J. (1971). Mental rotation of three-dimensional objects. Science, 171, 701-703.

Siddiqi, K., Tresness, K. J., \& Kimia, B. B. (1996). Parts of visual form: Psychophysical aspects. Perception, 25, 399-424.

TARR, M. J. (1995). Rotating objects to recognize them: A case study on the role of viewpoint dependency in the recognition of threedimensional objects. Psychonomic Bulletin \& Review, 2, 55-82.

TJAn, B. S., \& LegGe, G. E. (1998). The viewpoint complexity of an object-recognition task. Vision Research, 38, 2335-2350.

VAN LIER, R, \& WAGEMANS, J. (1997). Perceptual grouping measured by color assimilation: Regularity versus proximity. Acta Psychologica, 97, 37-70.

VAN LieR, R., \& Wagemans, J. (1998). Effects of physical connectivity on the representational unity of multi-part configurations. Cognition, 69, B1-B9.

Watson, S. E., \& Kramer, A. F. (1999). Object-based visual selective attention and perceptual organization. Perception \& Psychophysics, 61, 31-49.

Wertheimer, M. (1923). Untersuchungen zur Lehre von der Gestalt [Investigations for the use of the study of Gestalt]. Psychologische Forschung, 4, 301-350.

(Manuscript received July 31, 2001;

revision accepted for publication April 4, 2003.) 